\title{
Conservation success through IPBES-guided transformative change
}

Timothy C. Bonebrake ${ }^{1}$, Fengyi Guo ${ }^{1,2}$, Caroline Dingle ${ }^{1}$, David M. Baker ${ }^{1}$, Roger L. Kitching ${ }^{3}$, Louise A. Ashton ${ }^{1,3 *}$

${ }^{1}$ School of Biological Sciences, The University of Hong Kong, Pokfulam, Hong Kong

2 Department of Ecology and Evolutionary Biology, Princeton University, Princeton, NJ 08544, USA

${ }^{3}$ Environmental Futures Research Institute and School of Environment and Science, Griffith University, Nathan, QLD, Australia

*Correspondence : lashton@hku.hk (L.A.Ashton)

In response to our perspective on integrating proximal and horizon threats to biodiversity [1], Titeux et al. [2] objected to one of our sentences: "Titeux et al. [3] specifically highlight the Intergovernmental Platform on Biodiversity and Ecosystem Services (IPBES) as biased towards climate change research." To be more precise, Titeux et al. [3] stated in the abstract that "biodiversity scenarios mostly focus on the future impacts of climate change" and that "the current state of integration between ecological and land system sciences is leading to biased estimation of actual risks and therefore constrains the implementation of forward-looking policy responses to biodiversity decline." They then concluded the paper with a call: "IPBES offers a timely opportunity for taking up this challenge, but this independent body can only do so if adequate research efforts are undertaken." We appreciate that Titeux et al. [2] felt it necessary to correct our, admittedly, simplified summation of their work. However, we don't believe that this amounted to misrepresentation (though the reader can judge for themselves).

As highlighted by Titeux et al. [2], there is significant alignment in objectives between each of our perspectives and the IPBES Global Assessment [4]. Acknowledgment and active incorporation of multiple threats and scales into policy planning will be the only successful way forward. Upcoming additional IPBES reports [2] that further model/analyze interactions of biodiversity threats will undoubtedly advance conservation efforts broadly. Similarly, the recently released New Global Deal for Biodiversity (NGD) provides a blueprint for conserving biodiversity that explicitly integrates multiple threats [5]. The NGD proposes win-win strategies for protecting areas of habitat based on their classifications that include high diversity, connectivity and areas with high carbon-storage values, serving the multiple conservation goals of protecting biodiversity, maintaining ecosystem services and mitigating climate change [5].

But perhaps the strongest message from the IPBES Global Assessment [4] is the need for transformative change. Immediate and massive changes to global economic and political systems are needed in order to slow the deterioration of nature and biodiversity. Traditional conservation efforts have shown considerable successes, for example, in reversing declines of species or restoring ecosystems. Such interventions must continue and we hope that our framework for integrating proximal and horizon threats will lead to greater positive outcomes. However, it won't be enough. Without the transformative change championed by IPBES, ultimately we will fail to stop the decline and loss of millions of species. Humanity is resourceful though and we're optimistic that IPBES will be taken on-board by governments globally to take the first step in correcting damage done in recent centuries. This kind of transformative change 
would effectively counter both proximal and horizon threats to biodiversity and maintain nature's integrity for generations.

1 Bonebrake, T.C. et al. (2019) Integrating proximal and horizon threats to biodiversity for conservation. Trends Ecol. Evol. DOI: 10.1016/j.tree.2019.04.001

2 Titeux, N. et al. (2019) IPBES promotes integration of multiple threats to biodiversity. Trends Ecol. Evol.

3 Titeux, N. et al. (2016) Biodiversity scenarios neglect future land-use changes. Glob. Chang. Biol. 22, 2505-2515

4 IPBES (2019) Summary for policymakers of the global assessment report on biodiversity and ecosystem services of the Intergovernmental Science-Policy Platform on Biodiversity and Ecosystem Services, Advanced Unedited Edition, 6 May 2019, https://www.ipbes.net/global-assessment-biodiversity-ecosystem-services.

5 Dinerstein et al 2019. A Global Deal For Nature: Guiding principles, milestones, and targets. Science Advances 